\title{
Improving Walking-Robot Performances by Optimizing Leg Distribution
}

\author{
P. Gonzalez de Santos, E. Garcia and J. Estremera \\ Department of Automatic Control \\ Industrial Automation Institute - CSIC \\ Crta. Campo Real Km 0.2 \\ 28500 Arganda del Rey \\ Madrid, Spain, \\ phone +34918711900 \\ Fax: +34 918717050 \\ P. Gonzalez de Santos is the corresponding author \\ e-mail:pgds@iai.csic.es.
}




\title{
Improving Walking-Robot Performances by Optimizing Leg Distribution
}

\author{
P. Gonzalez de Santos, E. Garcia and J. Estremera \\ Department of Automatic Control \\ Industrial Automation Institute - CSIC \\ Crta. Campo Real Km 0.2 \\ 28500 Arganda del Rey \\ Madrid, Spain.
}

\begin{abstract}
Walking-robot technology has already achieved an important stage of development, as demonstrated in a few real applications. However, walking robots still need further improvement if they are to compete with traditional vehicles. A potential improvement could be made through optimization at design time. A better distribution of the legs around a robot's body can help decrease actuator size in the design procedure and reduce power consumption during walking as well, which is of vital importance in autonomous robots. This paper, thus, presents a method focused on the distribution of legs around the body to decrease maximum foot forces against the ground, which play heavily in determining robot shape and actuator size. Some experiments have been performed with the SILO6 walking robot to validate the theoretical results.
\end{abstract}

KEY WORDS: Energy efficiency, legged locomotion, mobile robots, optimization methods.

\section{Introduction}

Walking robots have been investigated and developed intensively by many universities, research centers, and companies since the late 1970's. Most of them are laboratory prototypes, but there are also a few walking machines built for specific applications that have achieved acceptable reliability, such as the Dante II (Bares and Wettergreen, 1999), built for volcano inspection, the Aquarobot, designed for underwater surveying of seawalls 
(Akizono et al. 1989), the ROWER, intended for naval construction (Gonzalez de Santos et al., 2000; 2002), and the Plustech walking machine, used for work in forests (Plustech-Oy, 2005).

Generally speaking, however, walking robots have many shortcomings that bar them from wider use in industry and services. For instance, legged robots are still heavy, bulky, very slow, and inefficient from the energy-expenditure point of view, which is a fundamental issue in autonomous robots. In other words, although legged robots have already demonstrated their capability to perform many tasks wheeled or tracked vehicles cannot handle, some features must still be improved before legged robots can meet present requirements for autonomous robots in industry and services.

So far the designers of real walking robots have focused on the selection of the number of legs, the kinematic study of static stability and the design of leg mechanisms. There are, however, other issues related with walking-machine design that have received more timid scrutiny. For instance, it is easy to infer that legs in the mammal configuration can support heavy masses, while the insect configuration provides better static stability. Nonetheless, configuration notwithstanding, leg distribution around the body could help in improving some robot features. For instance, walking robots usually have prismatic bodies on which electronic equipment is loaded and legs are attached (Bares and Wettergreen, 1999; (Nonami et al., 2000; Pfeiffer and Weidemann, 1991; Plustech-Oy, 2005; Salmi and Halme, 1996; Song, and Waldron, 1989); but body shape can determine static stability, as studied in (Schneider et al., 2000). Similarly, leg distribution around the body could also be adjusted to decrease maximum foot forces. This enables lower-torque actuators (that means smaller and lighter motor and/or smaller and lighter reducers) to be used to support the robot's weight, thereby reducing the weight, increasing the speed, or both at the same time.

All the legs on a walking robot are normally built on the same design, although mammals and insects possess very different rear and front (and sometimes middle) legs. The single standard leg design has many advantages in terms of design cost, replacements, modularity and so on. In quadrupeds leg distribution around the body is a simple thing, because not many combinations are available. Hexapods, on the other hand, offer a wider range of possibilities, as we will see below.

The purpose of this article is to contribute to the optimization of the design of walking 
robots by distributing identical legs around a hexapod body. In this distribution we determine the points in which the legs are fixed to the body to minimize the maximum exerted foot force. This minimization is achieved for the most common situation for a mobile robot i.e. the robot is moving on flat terrain, following a straight-line trajectory and performing a tripod gait. Thus, the design is first optimized for the most common scenario and then optimization for other possible scenarios would rely on defining new control algorithms, which is the next step in this research. The significance of this article's contribution relies on decreasing maximum foot forces in walking robots by factoring in design details and thus decreasing actuator size and also decreasing energy consumption during walking, which is a basic concern in autonomous vehicles. This aspect of legged locomotion has never been addressed before and is a previous step to design control algorithms that reduce power consumption during walking, which, as mentioned above, is the next step in this research.

This work focuses on the design of statically-stable walking robots and thus dynamic effects are not taken into account. However, the mass and positions of the legs are used to compute the $C O G$ of the robot.

This article is organized as follows: Section 1 introduces the needs of walking-robot design optimization. Section 2 presents the foot forces exerted by a hexapod performing an alternating-tripod gait. Next, Section 3 studies the influence of leg-body attachment points in the force-distribution problem. With this background, Section 4 presents a method for computing the points at which the legs should be attached to the body to minimize the maximum exerted foot force. Section 5 illustrates some experiments that have been carried out with the SILO6 walking robot (Gonzalez de Santos et al., 2005a; SILO6, 2006) to validate the theoretical results. Finally, we present some conclusions.

\section{Foot Forces along a Locomotion Cycle}

Statically-stable walking robots are intrinsically slow machines, and machine speed is well known to depend theoretically on the number of legs the machine has (Waldron et al., 1984). Hence, a hexapod can achieve higher speed than a quadruped, and a hexapod achieves its highest speed when using a wave gait with a duty factor of $\beta=1 / 2$, that is, using alternating tripods (Song and Waldron, 1989). Although the stability obtained by 
using alternating-tripod gaits is lower than the stability provided by other gaits which maintain four or five legs in support, alternating tripods are the most widely used by hexapods because of the speed they impart. "Alternating tripods" means that two nonadjacent legs on one side and the middle leg on the opposite side alternate in supporting the robot.

To analyze the foot forces that a walking robot must exert, let us take into account the following considerations. The robot consists of a body and six legs. The origin of the body reference frame $(x, y, z)$ is located at the body's geometric center (see Fig. 1). The body mass is $M_{B}$, and its center of gravity $C O G_{B}$ is fixed at $\left(x_{\mathrm{C}}, y_{\mathrm{C}}, z_{\mathrm{C}}\right)$. Each leg has a mass of $M_{L}$, and its center of gravity $C O G_{L}$ is located at $\left(x_{L_{i}}, y_{L_{i}}, z_{L_{i}}\right)$, which components vary with both the leg $i$ and the sampling instant $k$. The total weight of the robot is then $W=\left(M_{B}+6 M_{L}\right) g$. As the position of the $C O G_{L}$ changes with the foot positions, the center of gravity of the robot, $C O G$, varies too. In this model, all leg workspaces lie at the same distance with respect to the longitudinal axis of the body ( $x$-axis), and the body is level (see Fig. 1).

Let us assume that

$$
\tilde{\mathbf{F}}_{p q r}=\left(\mathbf{f}_{p}, \mathbf{f}_{q}, \mathbf{f}_{r}\right)^{T}
$$

is the foot-force vector when legs $p, q$, and $r$ are in support, where $\mathbf{f}_{i}=\left(f_{i_{x}}, f_{i_{y}}, f_{i_{z}}\right)^{T}$ is the ground-reaction force in foot $i$ (-f $\mathbf{f}_{i}$ is the force that foot $i$ must exert against the ground), and that the wrench $\tilde{\mathbf{W}}_{p q r}$ contains the forces $\left(\mathbf{F}_{\mathbf{x}}, \mathbf{F}_{\mathbf{y}}, \mathbf{F}_{\mathbf{z}}\right)$ and moments $\left(\mathbf{M}_{\mathbf{x}}, \mathbf{M}_{\mathbf{y}}, \mathbf{M}_{\mathbf{z}}\right)$ acting on the robot's $C O G$ and represents the robot's payload, any external applied load and the inertia effects of the robot's body (Jiang et al., 2001). That is

$$
\tilde{\mathbf{W}}_{p q r}=\left(\mathbf{F}_{\mathbf{x}}, \mathbf{F}_{\mathbf{y}}, \mathbf{F}_{\mathbf{z}}, \mathbf{M}_{\mathbf{x}}, \mathbf{M}_{\mathbf{y}}, \mathbf{M}_{\mathbf{z}}\right)^{T} .
$$

Under this condition, the equilibrium equations that balance forces and moments when three legs $(p, q$, and $r)$ are in their support phase can be obtained from Fig. 1 as 


$$
\begin{aligned}
& f_{p_{x}}+f_{q_{x}}+f_{r_{x}}+F_{x}=0 \\
& f_{p_{y}}+f_{q_{y}}+f_{r_{y}}+F_{y}=0 \\
& f_{p_{z}}+f_{q_{z}}+f_{r_{z}}+F_{z}=0 \\
& -z_{p} f_{p_{y}}+y_{p} f_{p_{z}}-z_{q} f_{q_{y}}+y_{q} f_{q_{z}}-z_{r} f_{r_{y}}+y_{r} f_{r_{z}}-z_{c} F_{y}+y_{c} F_{z}+M_{x}=0 \\
& z_{p} f_{p_{x}}-x_{p} f_{p_{z}}+z_{q} f_{q_{x}}-x_{q} f_{q_{z}}+z_{r} f_{r_{x}}-x_{r} f_{r_{z}}+z_{c} F_{x}-x_{c} F_{z}+M_{y}=0 \\
& -y_{p} f_{p_{x}}+x_{p} f_{p_{y}}-y_{q} f_{q_{x}}+x_{q} f_{q_{y}}-y_{r} f_{r_{x}}+x_{r} f_{r_{y}}-y_{c} F_{x}+x_{c} F_{y}+M_{z}=0
\end{aligned}
$$

These equations are normally written in matrix form as

$$
\tilde{\mathbf{A}}_{p q r} \tilde{\mathbf{F}}_{p q r}=-\tilde{\mathbf{B}}_{c} \tilde{\mathbf{W}}_{p q r}
$$

where

$$
\tilde{\mathbf{A}}_{p q r}=\left(\begin{array}{ccc}
\mathbf{I}_{\mathbf{3}} & \mathbf{I}_{\mathbf{3}} & \mathbf{I}_{\mathbf{3}} \\
\mathbf{R}_{p} & \mathbf{R}_{q} & \mathbf{R}_{r}
\end{array}\right)
$$

and

$$
\tilde{\mathbf{B}}_{c}=\left(\begin{array}{ll}
\mathbf{I}_{\mathbf{3}} & \mathbf{0}_{\mathbf{3}} \\
\mathbf{R}_{c} & \mathbf{I}_{\mathbf{3}}
\end{array}\right) .
$$

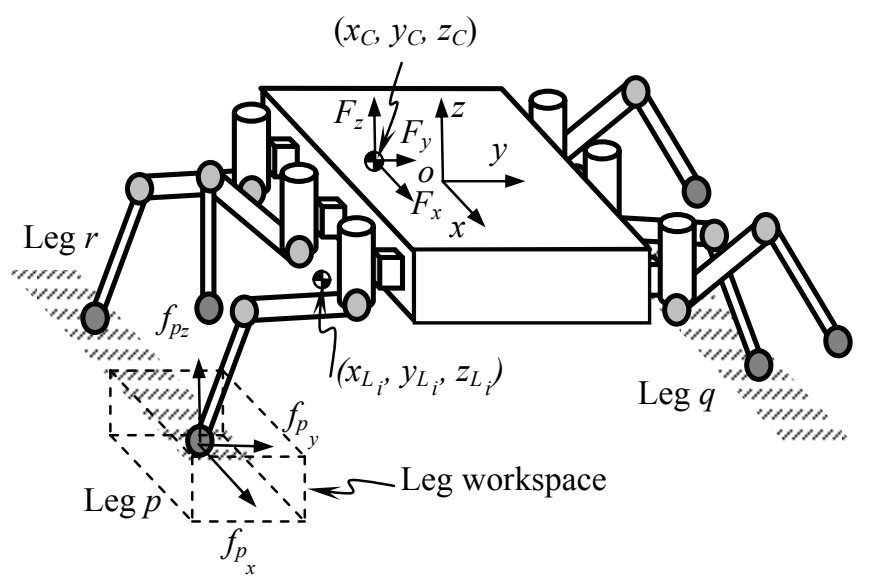

Fig. 1 Robot reference frame and forces acting on the robot 
$\mathbf{I}_{\mathbf{3}}$ is the $(3 \times 3)$ identity matrix, and $\mathbf{R}_{i}$ is the $(3 \times 3)$ skew symmetric matrix of the vector $\left(x_{i}, y_{i}, z_{i}\right)^{T}$ given by

$$
\mathbf{R}_{i}=\left(\begin{array}{ccc}
0 & -z_{i} & y_{i} \\
z_{i} & 0 & -x_{i} \\
-y_{i} & x_{i} & 0
\end{array}\right) .
$$

This matrix defines the position of foot $i(i=p, q, r)$ or the $C O G(i=c)$ in the robot's reference frame $(x, y, z)$ (see Fig. 2(a)).

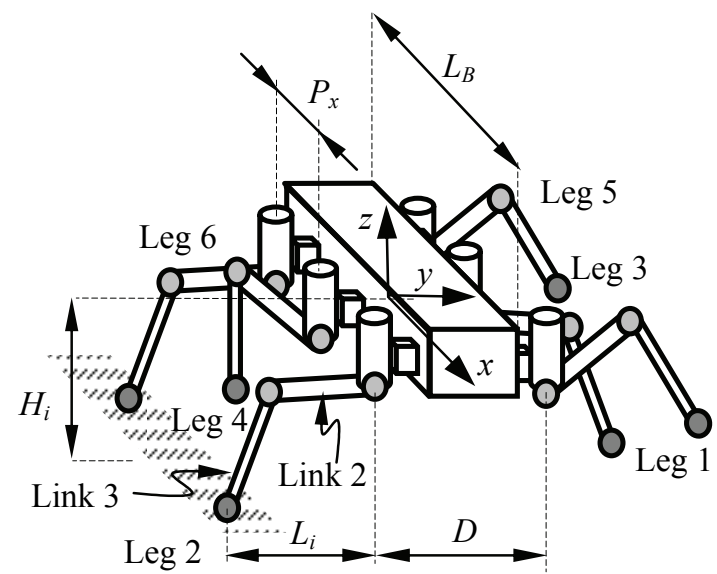

(a)

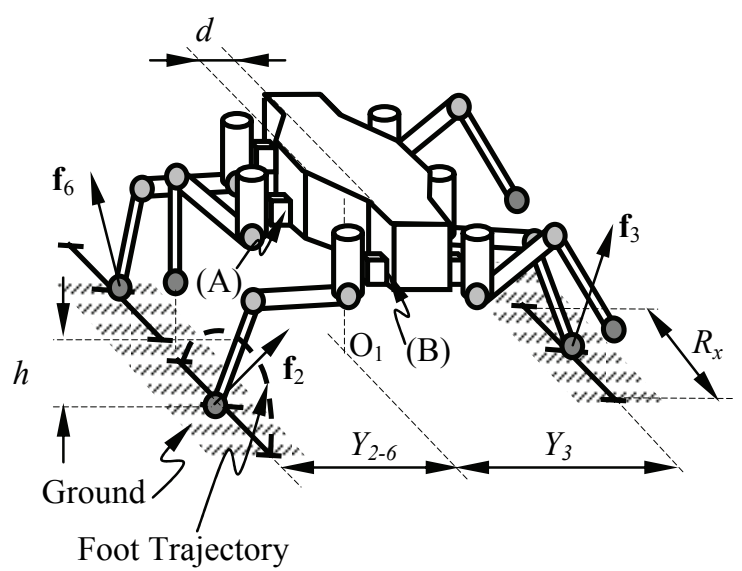

(b)

Fig. 2 Geometric models of the walking robot. 
For force-computation purposes, the robot is assumed to describe a continuous alternating-tripod gait (constant body speed) that consists of two main phases. In the first phase, legs 2, 3, and 6, for instance, are in support and moving backwards at a constant speed (continuous gait), while legs 1, 4, and 5 are in their transfer phase, moving forward to their next footholds (see Fig. 2(a) for leg definition). Each supporting foot follows a straight-line trajectory on the ground parallel to the trajectory of the other supporting feet.

The Euclidean distance between the centers of the workspaces of the adjacent legs on one side is termed the stroke pitch, $P_{x} \geq 0$ (see Fig. 2 for parameter definition). This is a fixed parameter that depends on the geometry of the walking robot and in this case coincides with the distance between adjacent-collateral leg reference frames (see Fig. 2).

Some additional geometric parameters are the Euclidean distance between the leg reference frames of non-collateral adjacent legs, $D \geq 0$, and the Euclidean distance between the point where a middle leg is attached to the body (A) and the point where a corner leg is attached to the body (B), along the direction of the $y$-axis, $d \geq 0$ (see Fig. 2(b)). Some variables that depend on the specific gait are the Euclidean distance from the trajectory of foot $i$ to the $z$-axis of the leg reference frame, $L_{i} \geq 0$; the Euclidean distance of the foot $i$ to the plane $x-y$ of the body reference frame, $H_{i} \geq 0$, i.e. $z_{i}=-H_{i}$; the step length (also known as leg stroke), $R_{x} \geq 0$; and the step height over the ground, $h \geq 0$. All these parameters and variables are defined in Fig. 2 and the relevant kinematic and mass parameters are given in Table 1.

The alternating-tripod gait algorithm computes the foot components at any time as (Gonzalez de Santos et al., 2005b)

$$
\left(x_{i}(k) \quad y_{i}(k) \quad z_{i}(k)\right)^{T}=G\left(k, i, R_{x}, L_{i}, H_{i}, h, d\right) ; \quad \text { for } i=1, . ., 6 ; k=1, \ldots, 2 N
$$

where $k$ is the sample period, $i$ is the leg number, $2 N$ is the number of samples in a locomotion cycle, and $G$ is the gait function whose components are

$$
x_{i}(k)=\sigma(i) P_{x}+(-1)^{\xi\left(\frac{k}{N+1}\right)} R_{x}\left(\frac{1}{2}-\frac{1}{N-1}(((k-1) \bmod N)-1)\right)
$$




$$
\begin{gathered}
y_{i}(k)=\left\{\begin{array}{cl}
(-1)^{i+1}\left(\frac{D}{2}+L_{i}\right) ; & \text { for } i=1,2,5,6 \\
(-1)^{i+1}\left(\frac{D}{2}+L_{i}+d\right) ; & \text { for } i=3,4
\end{array}\right. \\
z_{i}(k)= \begin{cases}h \sin \left(\frac{\pi k}{N}\right)\left(1-\xi\left(\frac{k}{N+1}\right)\right)-H_{i} ; \text { for } i=1,4,5 \\
-h \sin \left(\frac{\pi k}{N}\right) \xi\left(\frac{k}{N+1}\right)-H_{i} ; \text { for } i=2,3,6 .\end{cases}
\end{gathered}
$$

Function $\sigma(i)$ defines the displacement of the body attachment of leg $i$ with respect to the center of the body reference frame $(x, y, z)$ along the $x$-axis and is given by

$$
\sigma(i)=\left\{\begin{aligned}
1 & \text { for a front leg } \\
0 & \text { for a middle leg } \\
-1 & \text { for a rear leg }
\end{aligned}\right.
$$

$\xi(x)$ is the function which rounds the element $x$ to the nearest integer towards zero, and mod represents the function modulus.

$z_{i}$ equals $-H_{i}$ for a leg in the support phase and $z_{i}$ is greater than $-H_{i}$ for a leg in its transfer phase. The leg trajectory for the $z$-component can be defined in several very different ways: as a piecewise function, as a polynomial function, as a trigonometric function, and so on. For the sake of simplicity of formulation, a piecewise function has been chosen that consists of a straight segment and a sine function, and thus $z_{i}(k)$ is defined by (11), where $-H_{i}$ is the $z$-component of the foot $i$ that depends on the terrain the robot is stepping.

With these foot positions, the foot forces along a whole locomotion cycle can be computed from (4), which is an underdetermined system, because it consists of six equations and nine unknowns (the components of the three vectors $\mathbf{f}_{i}(3 \times 1)$ ) and its solutions can be found by using many different methods. The first and most popular method for solving the force distribution in closed kinematic chains is the pseudo-inverse, which gives the solution to (4) that minimizes the sum of the squares of the foot forces. This method is also known as the minimum norm solution and has been used intensively in the control of legged robots (Gorinevsky and Shneider, 1990; Klein and Chung, 1987; Klein and Kittivatcharapong, 1990; Kumar and Waldron, 1988; Kumar and Waldron, 
1990), More recently, some optimization methods have been proposed for solving the force-distribution problem that relay on criteria such as minimum force, load balance, safety margin or friction constraints (Chen et al., 1999; Zhou et al., 2000). These methods seem to be more practical than the pseudo-inverse method in terms of computation time. However, an alternative pseudo-inverse formulation has shown the pseudo-inverse method is good enough to solve the force-distribution problem (Jiang et al., 2001).

The pseudo-inverse method computes the foot forces of equation (4) as

$$
\tilde{\mathbf{F}}_{p q r}=-\tilde{\mathbf{A}}_{p q r}^{+} \tilde{\mathbf{B}}_{c} \tilde{\mathbf{W}}_{p q r}
$$

where matrix $\tilde{\mathbf{A}}_{p q r}^{+}$is the pseudo-inverse of $\tilde{\mathbf{A}}_{p q r}$ and is given by

$$
\tilde{\mathbf{A}}_{p q r}^{+}=\tilde{\mathbf{A}}_{p q r}^{T}\left(\tilde{\mathbf{A}}_{p q r} \tilde{\mathbf{A}}_{p q r}^{T}\right)^{-\mathbf{1}} .
$$

The solution of (13) at the instant $k$ for the first half-locomotion cycle of the alternatingtripod gait when the body is level and moving at constant speed (no external forces acting on the $C O G)$ is

$$
\left(\begin{array}{r}
\mathbf{f}_{1}(k) \\
\mathbf{f}_{4}(k) \\
\mathbf{f}_{5}(k)
\end{array}\right)=-\tilde{\mathbf{A}}_{145}^{+}(k) \tilde{\mathbf{B}}_{c}(k)\left(\begin{array}{c}
0 \\
0 \\
-W \\
0 \\
0 \\
0
\end{array}\right)
$$

for legs in their support phase (legs 1, 4, and 5 along the first half-cycle) and

$$
\left(\begin{array}{l}
\mathbf{f}_{2}(k) \\
\mathbf{f}_{3}(k) \\
\mathbf{f}_{6}(k)
\end{array}\right)=\left(\begin{array}{l}
\mathbf{0} \\
\mathbf{0} \\
\mathbf{0}
\end{array}\right)
$$

for legs in their transfer phase (legs 2, 3, and 6 along the first half-cycle). The solution for the second half-cycle can be computed similarly, and the closed solution along the whole locomotion cycle can be written as 
Table 1. Main SILO6 robot features.

\begin{tabular}{|c|c|c|c|}
\hline \multirow{7}{*}{ Body } & \multirow{5}{*}{ Dimensions (m) } & Length $\left(L_{B}\right)$ & 0.88 \\
\hline & & Front/rear Width $(D)$ & 0.38 \\
\hline & & Middle Width & 0.63 \\
\hline & & Height & 0.26 \\
\hline & & Stroke Pitch $\left(P_{x}\right)$ & 0.365 \\
\hline & \multicolumn{2}{|l|}{ Mass (kg) } & $\approx 28.2 *$ \\
\hline & \multicolumn{2}{|l|}{ Speed $(\mathrm{m} / \mathrm{s})$} & 0.05 \\
\hline \multirow{10}{*}{ Leg } & \multirow{3}{*}{ Link Length (m) } & $a_{1}$ & 0.084 \\
\hline & & $a_{2}$ & 0.250 \\
\hline & & $a_{3}$ & 0.250 \\
\hline & \multirow{3}{*}{$\begin{array}{l}C O G_{L}(\mathrm{~m}) \\
\text { (with respect to }\left(X_{0}, Y_{0}, Z_{0}\right) \\
\text { when } \theta_{I}=90^{\circ} \text { and } \theta_{2}=0^{\circ} \text {, see } \\
\text { Fig. 10) }\end{array}$} & $X_{0}$ & 0 \\
\hline & & $Y_{0}$ & 0.105 \\
\hline & & $Z_{0}$ & 0.012 \\
\hline & Stroke $\left(R_{x}\right)(\mathrm{m})$ & \multicolumn{2}{|c|}{0.25} \\
\hline & Mass $(\mathrm{kg})$ & \multicolumn{2}{|c|}{$\approx 4.3^{*}$} \\
\hline & \multirow{2}{*}{ Foot Speed $(\mathrm{m} / \mathrm{s})$} & Transfer Phase & 0.140 \\
\hline & & Support Phase & 0.05 \\
\hline Robot & Total Mass (kg) & 54 & \\
\hline
\end{tabular}

*) Some parts of the leg, which are fixed to the body and do not move with the leg joints, are considered part of the body for simulation and control purposes. The whole leg, as illustrated in Fig. 10, weighs $5.4 \mathrm{~kg}$.

$$
\begin{gathered}
\left(\begin{array}{c}
\mathbf{f}_{1}(k) \\
\mathbf{f}_{4}(k) \\
\mathbf{f}_{5}(k)
\end{array}\right)=-\left(1-\xi\left(\frac{k}{N+1}\right)\right) \tilde{\mathbf{A}}_{145}^{+}(k) \tilde{\mathbf{B}}_{c}(k)\left(\begin{array}{c}
0 \\
0 \\
-W \\
0 \\
0 \\
0
\end{array}\right) \text { for } k=1, . ., 2 N \\
\left(\begin{array}{c}
\mathbf{f}_{\mathbf{2}}(k) \\
\mathbf{f}_{\mathbf{3}}(k) \\
\mathbf{f}_{\mathbf{6}}(k)
\end{array}\right)=-\xi\left(\frac{k}{N+1}\right) \tilde{\mathbf{A}}_{236}^{+}(k) \tilde{\mathbf{B}}_{c}(k)\left(\begin{array}{c}
0 \\
0 \\
-W \\
0 \\
0 \\
0
\end{array}\right) \quad \text { for } k=1, . ., 2 N .
\end{gathered}
$$


Equation (17) for the foot positions $G\left(k, i, R_{x}, L_{i}, H_{i}, h, d\right)$ defined by (8), assuming that the $C O G_{B}$ is at the origin of the body reference frame and considering the parameters used for ordinary walking: $R_{x}=0.25 \mathrm{~m}, L_{i}=0.334 \mathrm{~m}$ and $H_{i}=0.25 \mathrm{~m}$ for all $i, h=0.15 \mathrm{~m}$ and $d$ $=0$, yields the vertical foot-reaction forces depicted in Fig. 3. Note that the $C O G_{B}$ depends on the masses and positions of the different parts onboard (electronic equipment and subsystems), which can be easily located to make the $C O G_{B}$ coincident with the origin of the body reference frame. In this example, the locomotion cycle is 20 seconds, and the sample period is 0.2 seconds.

The horizontal foot forces obtained from (17) are null along the whole locomotion cycle. This guarantees that the pseudo-inverse solution is adequate for the theoretical case.

Fig. 3 shows how the force distribution is symmetrical, in the sense that the left and right legs exert the same force (although in different half-cycles) when they are in support. Also, the front and rear legs complement each other in force, such that the sum of the vertical forces of legs in the same tripod at any given time equals the weight of the robot, approximately $529.74 \mathrm{~N}$ in our example. The maximum foot force for each leg is indicated in the upper right corner of the corresponding leg plot in Fig. 3. We can observe that the middle legs must exert a force of up to $264.87 \mathrm{~N}$, while the corner legs only need to exert up to $223.14 \mathrm{~N}$. If all the robot legs are based on the same design, this design should focus on the middle legs' requirements, and as a result the corner legs will be over-sized. However, the vertical force a leg must exert or support depends heavily on that leg's foot position relative to the foot position of every other leg. Therefore, distributing legs around the body advantageously can help in properly distributing the foot forces among all the legs. Note that foot forces depend on foot positions, not body height. The section below discusses the leg-distribution problem. It is worthwhile to remark that $\mathbf{R}_{C}$ has been computed along a locomotion cycle taking into consideration the contribution of the moving center of gravity of the legs $\left(C O G_{L}\right)$. However, because of the symmetry of leg motions when using alternating tripods, the influence of the variation of the $C O G_{L}$ position is insignificant. A simplified study reported in (Gonzalez de Santos et al., 2005b), which considers the $C O G$ located at the center of the body, provides force shapes similar to that provided in Section 3 below. It has been computed that in this case study the $C O G$ deviates 
from the origin of the body reference frame by about $3 \mathrm{~mm}$ in the horizontal plane, and about $7.81 \mathrm{~mm}$ in the vertical plane, along a locomotion cycle. Nevertheless the optimization is performed with the body level and then the vertical position of the COG has not influence in the force distribution problem.

\section{Leg Distribution around the Robot's body}

When a legged robot is supported by a tripod, as in Fig. 2, the middle leg in its support phase for a given foot position is carrying about half the robot's weight, while the two collateral legs in their support phase are carrying about one-fourth of the robot's weight. This can be observed in Fig. 3 for $t=5$ seconds (legs 1, 4, and 5 in the support phase) and $t$ $=15$ seconds (legs 2, 3, and 6 in the support phase). These points correspond to the instants when the legs in question are in the middle of their workspaces, that is, when link 2 is perpendicular to the $x$-axis of the body. This circumstance is especially significant in traditional hexapod configurations, where all legs are placed at the same distance from the longitudinal axis of the robot's body.

Satisfactory force distribution and system homogenization can be achieved by shifting the middle legs' foot positions slightly from the body's longitudinal axis so that the middle legs support less weight and the corner legs increase their contribution to supporting the body.

The condition for sharing the weight of the robot evenly among the supporting legs for the case described above is given by (13) when the desired foot reaction forces are

$$
\mathbf{f}_{i}=\left(0,0, \frac{W}{3}\right)^{T} ; \text { for } i=2,3,6
$$

where $W$ is the total weight of the robot. Hence, $\tilde{\mathbf{F}}$ becomes

$$
\tilde{\mathbf{F}}_{236}=\left(0,0, \frac{W}{3}, 0,0, \frac{W}{3}, 0,0, \frac{W}{3}\right)^{T} .
$$

If we want the robot to be stationary with no external forces acting on it, then the total wrench $\tilde{\mathbf{W}}$ must be

$$
\tilde{\mathbf{W}}_{236}=(0,0,-W, 0,0,0)^{T} .
$$

That is, every foot in support phase $(2,3$, and 6$)$ is supposed to exert a vertical force of 
$W / 3$, while feet 2,3 , and 6 are at locations $\left(P_{X},-Y_{2-6},-H\right),\left(0, Y_{3},-H\right)$, and $\left(-P_{X},-Y_{2-6},-H\right)$, respectively (the definition of these parameters is indicated in Fig. 2(a)); thus matrix $\tilde{\mathbf{A}}_{236}$ becomes

$$
\tilde{\mathbf{A}}_{236}=\left(\begin{array}{ccccccccc}
1 & 0 & 0 & 1 & 0 & 0 & 1 & 0 & 0 \\
0 & 1 & 0 & 0 & 1 & 0 & 0 & 1 & 0 \\
0 & 0 & 1 & 0 & 0 & 1 & 0 & 0 & 1 \\
0 & H & -Y_{2-6} & 0 & H & Y_{3} & 0 & H & -Y_{2-6} \\
-H & 0 & -X_{2-6} & -H & 0 & 0 & -H & 0 & X_{2-6} \\
Y_{2-6} & X_{2-6} & 0 & -Y_{3} & 0 & 0 & Y_{2-6} & -X_{2-6} & 0
\end{array}\right)
$$

and $\tilde{\mathbf{B}}_{C}=\mathbf{I}_{6}$.
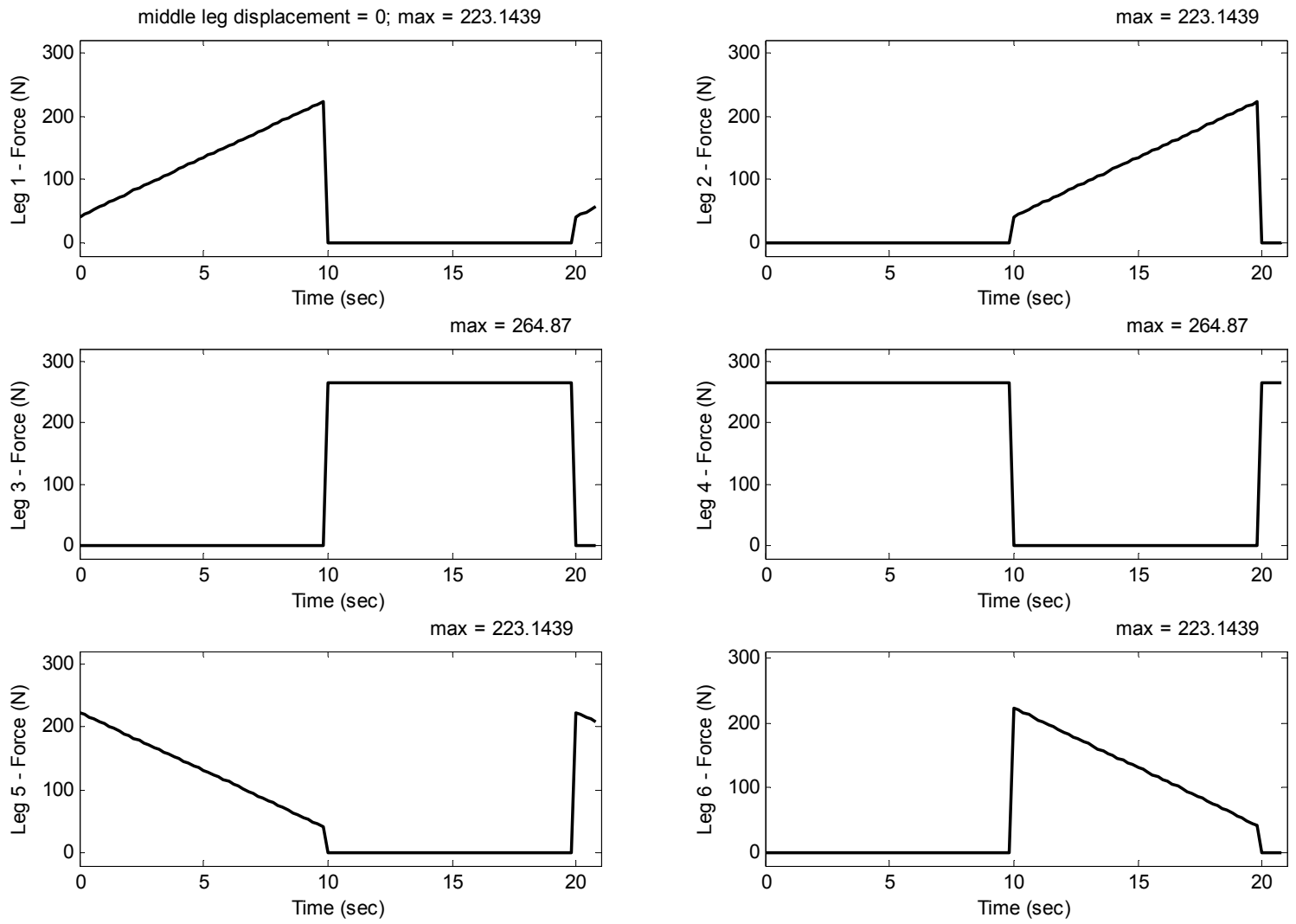

Fig. 3 Foot-reaction forces for one locomotion cycle of the alternating-tripod gait. 
Substituting (19), (20) and (21) into (4) yields

$$
\left(\begin{array}{c}
0 \\
0 \\
W \\
-\frac{2}{3} W Y_{26}+\frac{1}{3} W Y_{3} \\
0 \\
0
\end{array}\right)=-\left(\begin{array}{c}
0 \\
0 \\
-W \\
0 \\
0 \\
0
\end{array}\right)
$$

Equation (22) is satisfied if an only if

$$
Y_{3}=2 Y_{2-6} \text {. }
$$

That means the middle legs' feet, for this specific case, must be placed twice the distance from the longitudinal axis of the body as the corner legs' feet. Possible leg configurations are shown in Fig. 4. Configuration (a) resembles an insect configuration, but the legattachment points are at the same distance from the $x$-axis, that is $d=0$, and we can see that, to satisfy (23), the corner legs must follow trajectories very close to the body and about the boundaries of the workspace. Leg poses in this case do not appear natural. Configuration (b) resembles the crab configuration. In this case, leg-attachment points are at different distances from the $x$-axis, and every foot follows a similar trajectory that falls about the middle of the foot workspace ( $y$-axis). In this case, the leg can make a better use of its workspace.

Equation (23) gives the solution just for the specific robot pose in which footholds are symmetrical, as in the examples in Fig. 4; however, solutions should be found along a whole locomotion cycle, and the attachment point of the central legs should be moved so that the maximum foot force in any middle leg equals the maximum foot force in any corner leg. In this case, the legs can be designed to exert as little force against the ground as possible, thus helping to make the robot lighter and faster. That is, if foot forces are lower, then actuators are smaller or the required gearings are lower, which means higher speed and better mechanical efficiency. This helps in saving energy. Note that equation (10) has been parameterized by $d$ just to allow the following study. 


\section{Optimizing Leg Distribution around the Robot's body}

One possible solution for reducing foot forces as pointed out in Section 3 consists in using the configuration in Fig. 4(b) and computing the middle-leg displacement, $d$, that equals the maximum vertical force exerted in every leg along a locomotion cycle. For that, it is necessary to recalculate the foot forces for every foot position along a locomotion cycle. Then, the middle-leg displacement can be calculated and used, for instance, to eliminate the difference of the maximum vertical foot forces between pairs of legs. The procedure is as follows:

The foot positions for generating an alternating-tripod gait are now given by $G(k, i, 0.25,0.334,0.25,0.15, d)$ (see (8)). Note that in this case the parameter $d$ is a variable, because we want to compute the foot forces given by (17) along a locomotion cycle as a function of parameter $d$. Therefore, by varying parameter $d$, we can match the maximum force exerted by each foot along a locomotion cycle. The condition can be stated as

$$
\Phi(d)=\left(\begin{array}{c}
\max _{\forall k}\left(F_{z_{1}}(k, d)\right)-\max _{\forall k}\left(F_{z_{2}}(k, d)\right) \\
\max _{\forall k}\left(F_{z_{2}}(k, d)\right)-\max _{\forall k}\left(F_{z_{3}}(k, d)\right) \\
\max _{\forall k}\left(F_{z_{3}}(k, d)\right)-\max _{\forall k}\left(F_{z_{4}}(k, d)\right) \\
\max _{\forall k}\left(F_{z_{4}}(k, d)\right)-\max _{\forall k}\left(F_{z_{5}}(k, d)\right) \\
\max _{\forall k}\left(F_{z_{5}}(k, d)\right)-\max _{\forall k}\left(F_{z_{6}}(k, d)\right)
\end{array}\right)=\mathbf{0}_{5 \times 1} .
$$

The problem is then reduced to finding the parameter $d$ that yields

$$
\Phi(d)=\mathbf{0}_{5 \times 1} .
$$

In other words, we move the point where the middle legs ( 3 and 4 ) are attached to the body until the maximum foot force in all the legs equal. 
The solution of a general non-linear equation, such as $\Phi$, is efficiently provided by the Gauss-Newton method (Grace, 1994). This method requires an initial estimation of the solution and stops when it finds a local solution. For the geometric and mass parameters used in this study (see Table 1), and for foot trajectories at a distance $L_{i}=0.334 \mathrm{~m}$ from the longitudinal body axis, a step length $R_{x}=0.25 \mathrm{~m}$ and the trajectories of feet in transfer defined by $H_{i}=0.25$ and $h=0.15$ (see (9)-(11), function $\Phi$ vanishes for $d=0.123 \mathrm{~m}$, which gives a maximum foot-force value in all legs of $F_{\max }=237.05$ N. Fig. 5 plots the foot forces for the left legs. As mentioned above, the foot forces for the left and right legs are

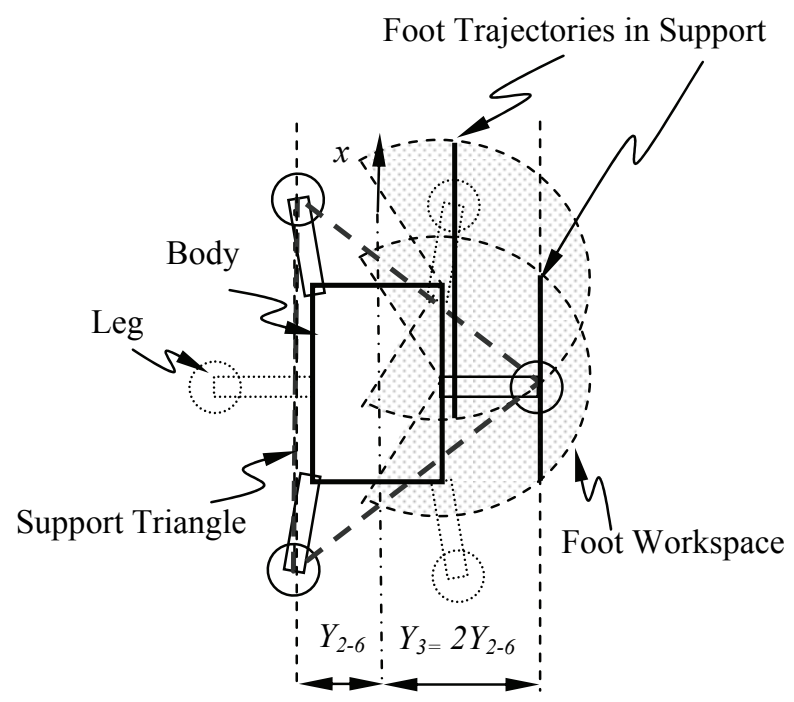

(a)

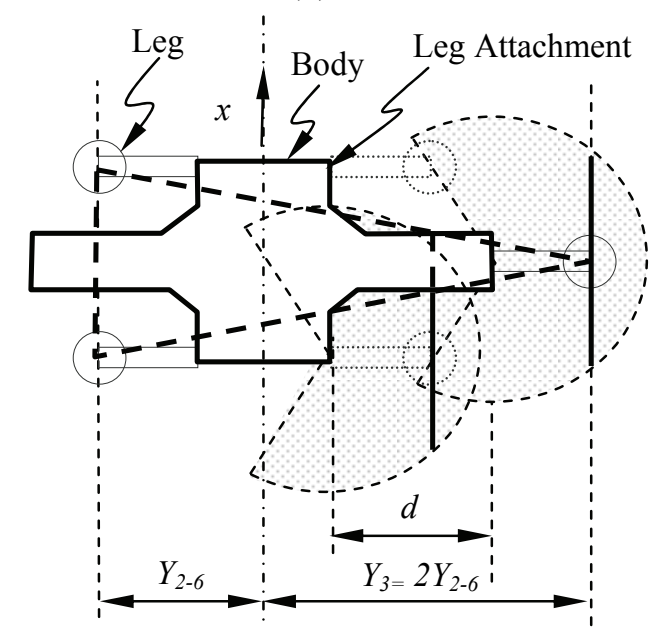

(b)

Fig. 4 Top view of two possible leg configurations: (a) insect, (b) crab. 

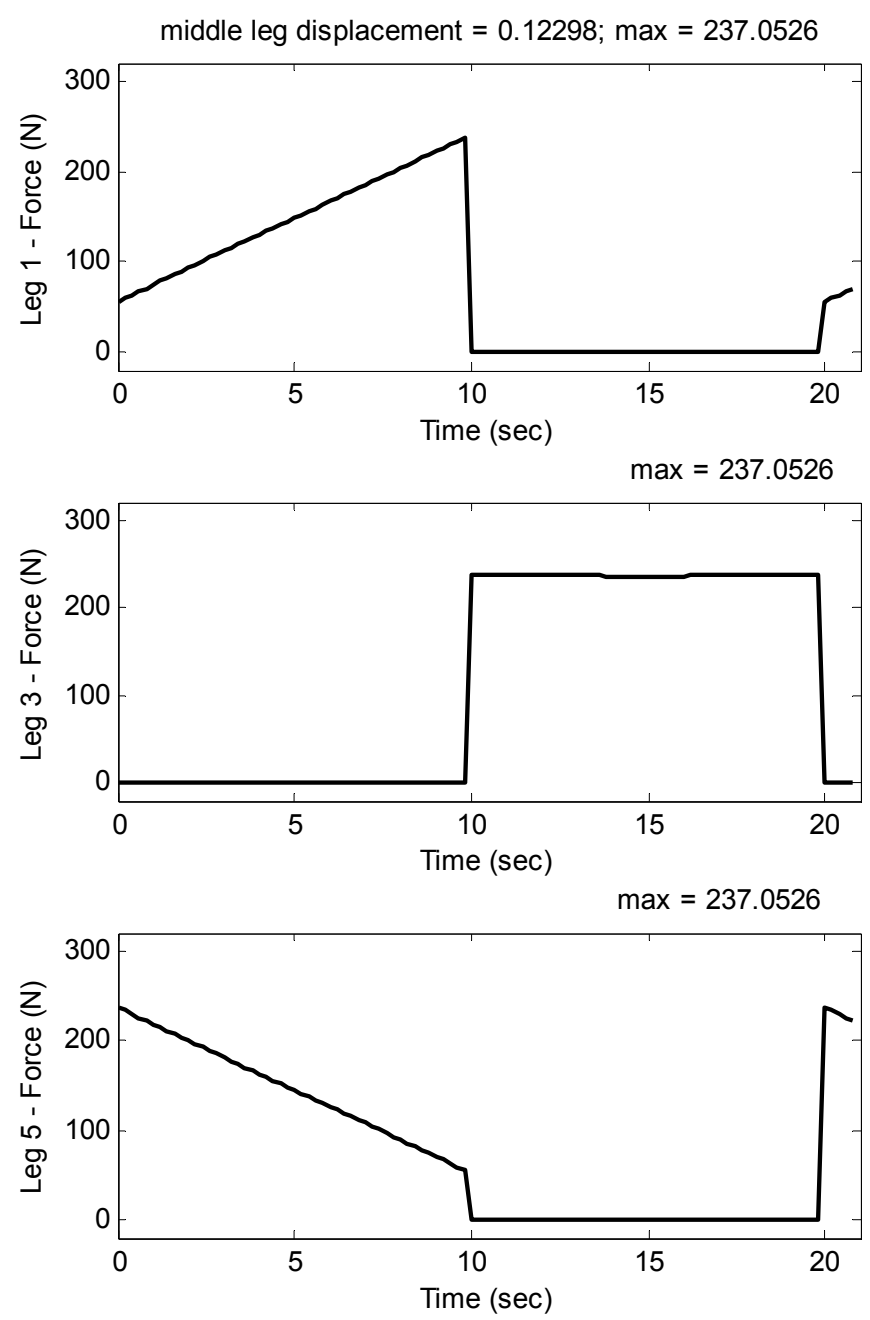

Fig. 5 Foot-reaction forces in the left legs for one locomotion cycle of an alternatingtripod gait when the middle legs are shifted by $0.123 \mathrm{~m}$.

symmetrical, so, for the sake of article length, the right legs' foot forces have not been plotted.

Therefore, by moving the point where the middle leg is attached to the body about $d=$ $0.123 \mathrm{~m}$, we can reduce the maximum exerted foot forces by $27.83 \mathrm{~N}$ (from $264.87 \mathrm{~N}$ to $237.04 \mathrm{~N}$ ) and thus reduce the maximum force by about $10.51 \%$. This feature also influences actuator/gear-set selection and the robot's general features, especially speed and power consumption as we will see below.

Note that this result does not depend on the robot's height, but on the foot trajectories; therefore, parameter $d$ has been computed for the most ordinary robot pose, $L_{i}=0.334 \mathrm{~m}$. 


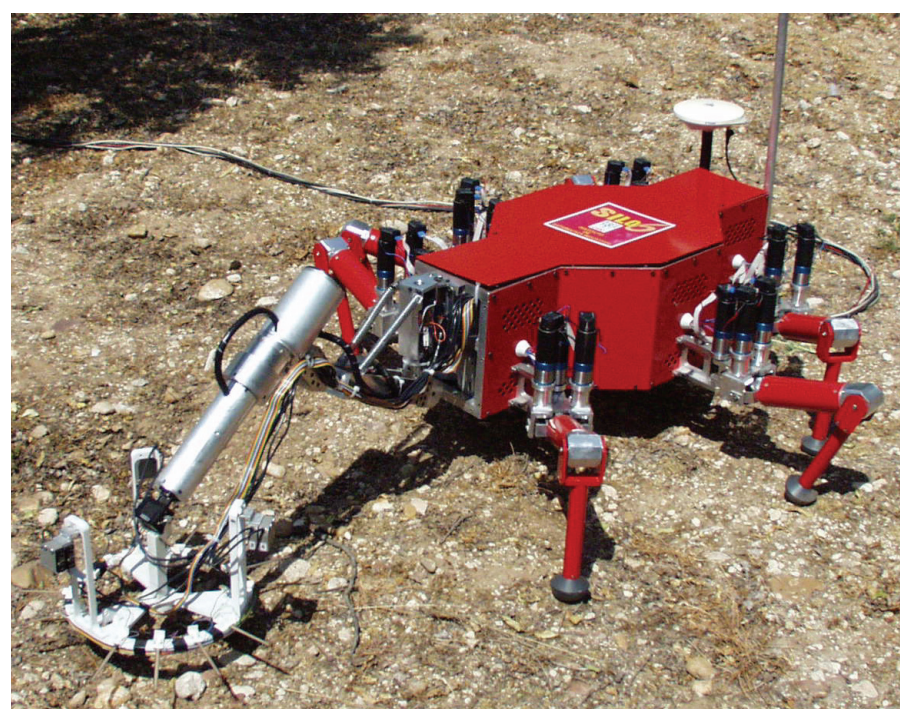

Fig. 6 The SILO6 walking robot.

In this pose, a foothold lies about the center of the foot's workspace.

These findings are especially significant for robots based on orthogonal legs (Bares, and Whittaker, 1993) in which vertical foot force is applied directly by an independent actuator; when maximum vertical support forces are minimized, actuators with lower power/torque or lower reduction gears can be used, thus decreasing weight and improving mechanical efficiency, which is a way of decreasing energy expenditure.

Fig. 3, which was obtained for $d=0$, shows how the theoretical foot forces exhibit some symmetry: all four corner legs have the same maximum foot force, and the two middle legs also exert an equal maximum force. Based on this property of force symmetry, the cost function (24) can be simplified to

$$
\Phi(d)=\max _{\forall k}\left(F_{z_{l}}(k, d)\right)-\max _{\forall k}\left(F_{z_{3}}(k, d)\right) .
$$

That is, we look for a value of $d$ that makes equal the maximum foot reaction forces of a corner leg and a middle leg; all other leg values equal each other because of the property of force symmetry. Equation (26) gives the same solution that (24), but it requires less computational burden.

\section{Experimental results}

The theoretical results presented in Section 4 above have been the basis of the design of the SILO6 walking robot (SILO6, 2006). This robot, shown in Fig. 6, has been built as the 
mobile platform of a system for detecting and locating antipersonnel landmines. The legs have been attached to the body at points that equal the maximum foot forces required to support the robot.

Some experiments have been conducted to validate the robot design, focusing on

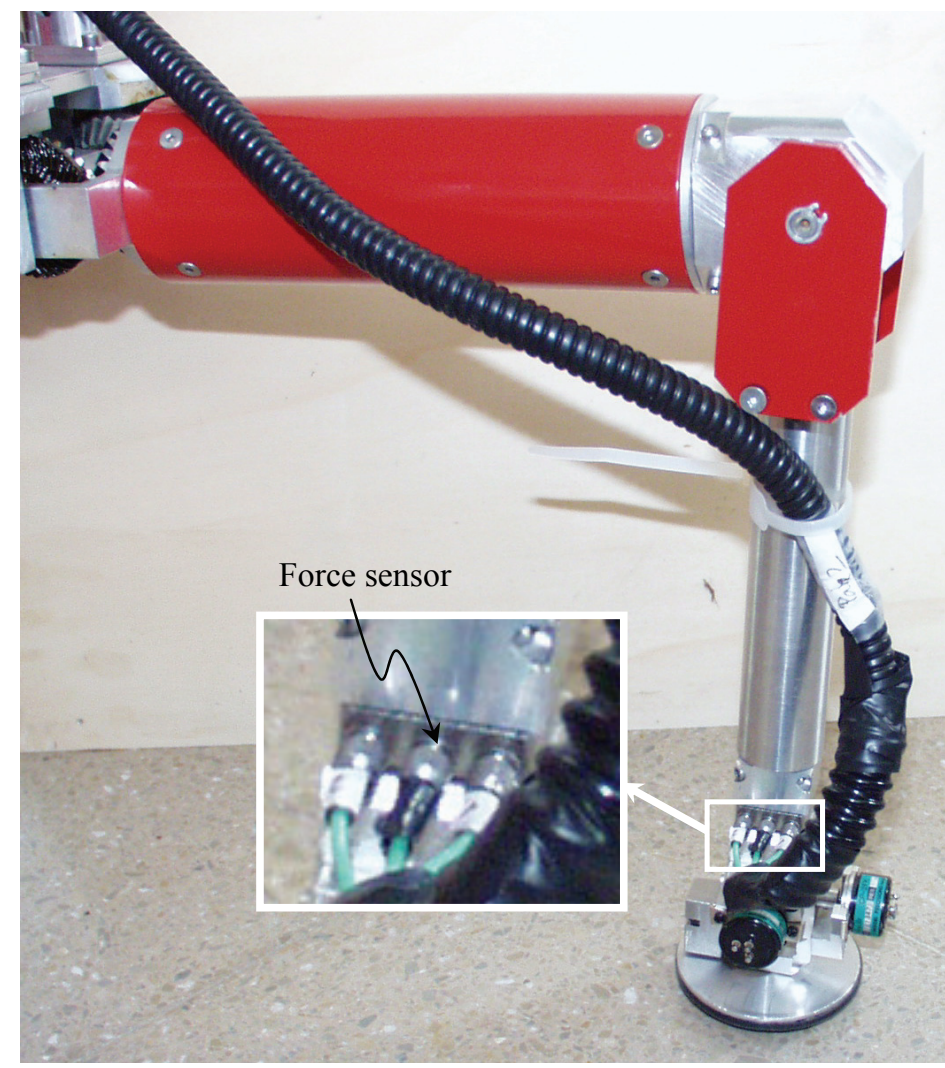

Fig. 7 Foot used in the experimental setup

measuring the foot forces along a locomotion cycle. For this purpose, every leg has been endowed with a foot that contains a quartz force transducer capable of measuring three orthogonal components of forces acting in an arbitrary direction (see Fig. 7). 
Foot forces are read from the transducers, conveniently filtered and expressed in the body reference frame by using the kinematic relationships included in Appendix 1. Fig. 8 plots the foot forces for the legs in support phase as well as the robot's total weight, which is computed by adding up the individual vertical foot forces. The average weight measured in this way is about $574.29 \mathrm{~N}$, while the real weight is $529.74 \mathrm{~N}$. This results in an error of about $7.76 \%$ in the measurement process. According to the results in Section 4, if the robot's $C O G$ is at the origin of the body reference frame, then maximum foot forces should be equal. In the real experiment, the robots $C O G$ is at $\left(x_{C O G}, y_{C O G}, z_{C O G}\right)=(-0.04 \mathrm{~m}, 0.0 \mathrm{~m}$, $0.03 \mathrm{~m}$ ). This negative displacement of the $C O G$ along the $x$-axis causes the rear legs to increase foot forces and the front legs to decrease foot forces. Fig. 9 illustrates this effect. Foot forces are computed as in Fig. 5, but the position of the robot's $C O G$ is changed to the new value. Note that after the leg support and for $t<2 \mathrm{~s}$ there is a transitory response because of the effects of foot accommodation, link flexion and joint backlash. These effects

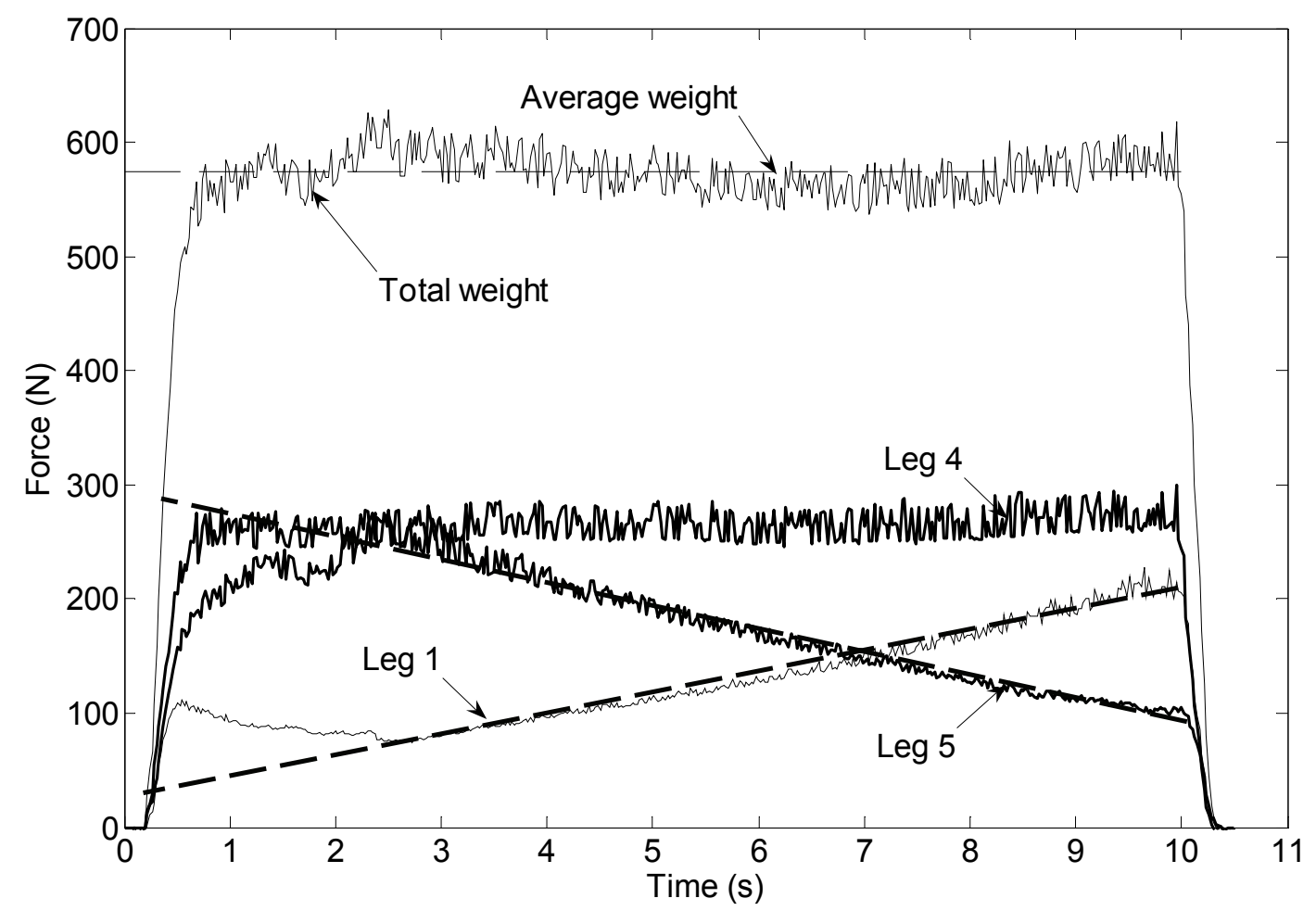

Fig. 8 Foot forces in the legs of the first tripod of the SILO6 walking robot along a half locomotion cycle 
change the behavior indicated by the simulation results, because no model for these effects has been considered. For $t>2 \mathrm{~s}$, the behavior is quite linear, as expected, and the foot liftoff does not present transitory performance because foot and joint accommodation do not take place in this type of event. The expected foot forces for legs 1 and 5 are drawn in a dashed line over the real foot forces in Fig. 8. Those are the expected results if no footground accommodation effects appear.

The experimental result also shows the tendency for the maximum foot forces to decrease in leg 1 and for the foot forces in leg 5 to increase, as indicated in Fig. 9. The errors lie within the range of the measurement errors (about $7.76 \%$ ).
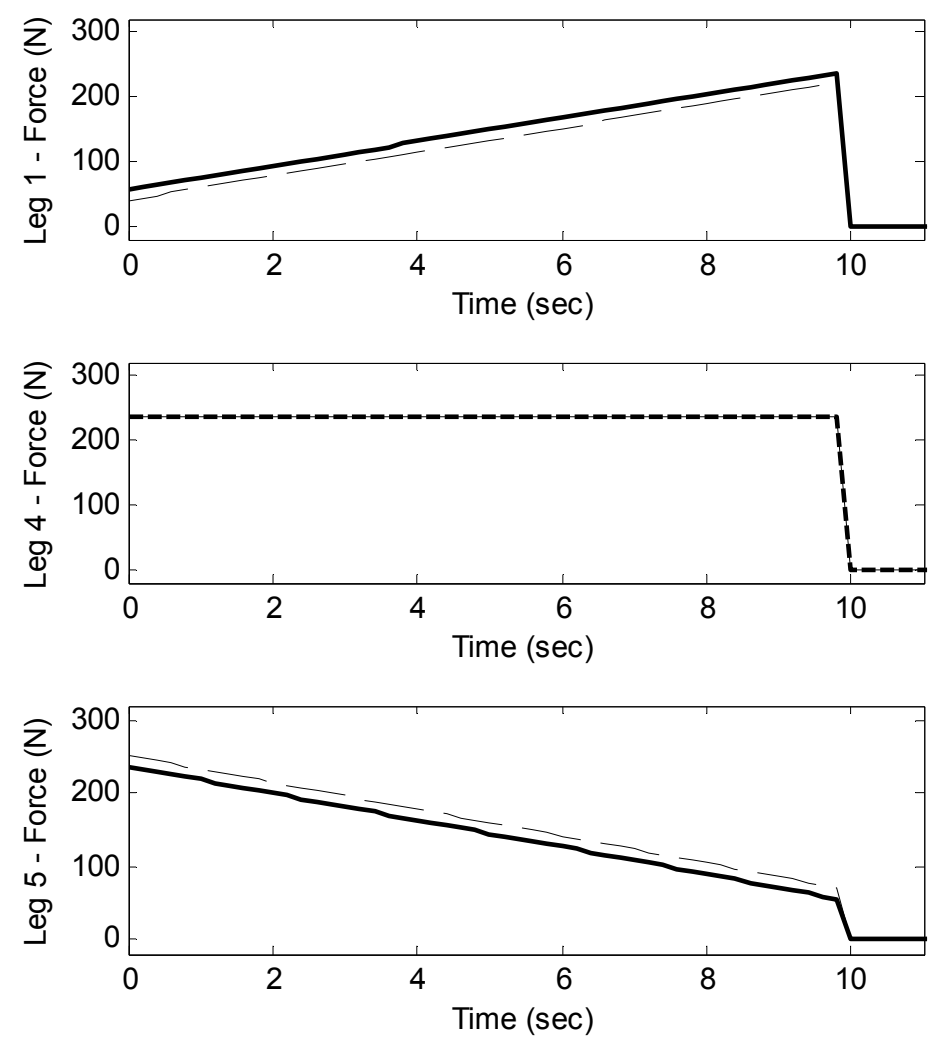

Fig. 9 Foot forces for the first tripod along a half locomotion cycle. The solution for $\left(x_{C O G}\right.$, $\left.y_{C O G}, z_{C O G}\right)=(0,0,0)$ is in a solid line. The solution for $\left(x_{C O G}, y_{C O G}, z_{C O G}\right)=(-0.04 \mathrm{~m}, 0.0$ $\mathrm{m}, 0.03 \mathrm{~m})$ is in a dashed line. 


\section{Conclusions}

So far, walking-robot development has sought only to explore the potential features of walking robots. It is now high time to start optimizing robot design and control methods to make these vehicles competitive with their wheeled or tracked counterparts. This paper proposes a way of improving robot features, in terms of using lower power/torque actuators (light-weighted actuators) and decreasing energy expenditure. The method consists in decreasing the maximum foot forces that a legged robot requires to support itself, by locating the legs around the robot's body in a way that enables the robot to use either lower power/torque actuators or lower reduction rates, and thus to gain speed. Our study shows that by displacing the middle legs in our hexapod by about $d=0.123 \mathrm{~m}$, the maximum supporting-foot forces can be reduced by 10.5\%. Traditional, well-known hexapod robots use prismatic bodies only and miss out on the advantages presented in this paper. This paper's authors were encouraged for that reason to build the SILO6 walking robot with the body shape shown in Fig. 2(b) and Fig. 6, which separates the middle legs' attachment points from the corner legs' attachment points, splitting along the $y$-axis.

Leg-force and joint-torque minimization are basic issues for walking-robot design and power optimization. Thus, further work shall be devoted to minimizing joint torques along a locomotion cycle, as a further step towards power consumption optimization.

\section{Appendix 1}

This appendix presents the leg kinematics used for simulation and control purposes. The direct kinematic relationships consist in envisioning the position $\left(x_{i}, y_{i}, z_{i}\right)^{T}$ of foot $i$ as a function of the joint components $\left(\theta_{i 1}, \theta_{i 2}, \theta_{i 3}\right)^{T}$. That is

$$
\left(x_{i}, y_{i}, z_{i}\right)^{\mathrm{T}}=\mathrm{T}_{D I R}\left(\theta_{\mathrm{i} 1}, \theta_{\mathrm{i} 2}, \theta_{\mathrm{i} 3}\right) ;
$$

where

$$
\mathrm{T}_{D I R}=\left(\begin{array}{c}
\cos \theta_{i 1}\left(a_{3} \cos \left(\theta_{i 2}+\theta_{i 3}\right)+a_{2} \cos \theta_{i 2}+a_{1}\right) \\
\sin \theta_{i 1}\left(a_{3} \cos \left(\theta_{i 2}+\theta_{i 3}\right)+a_{2} \cos \theta_{i 2}+a_{1}\right) \\
a_{3} \sin \left(\theta_{i 2}+\theta_{i 3}\right)+a_{2} \sin \theta_{i 2}
\end{array}\right)
$$


The inverse kinematics is defined by,

$$
\left(\begin{array}{lll}
\theta_{i 1} & \theta_{i 2} & \theta_{i 3}
\end{array}\right)^{T}=\mathbf{T}_{I N V}\left(x_{i}, y_{i}, z_{i}\right) ; \text { for } i=p, q, r
$$

where

$$
\mathrm{T}_{I N V}=\left(\begin{array}{c}
\operatorname{arctg}\left(\frac{y_{i}}{x_{i}}\right) \\
-\operatorname{arctg}\left(\frac{B_{i}}{A_{i}}\right)+\operatorname{arctg}\left(\frac{D_{i}}{ \pm \sqrt{A_{i}^{2}+B_{i}^{2}-D_{i}^{2}}}\right) \\
\operatorname{arctg}\left(\frac{z_{i}-a_{2} \sin \theta_{i 2}}{x_{i} \cos \theta_{i 1}+y_{i} \sin \theta_{i 1}-a_{2} \cos \theta_{i 2}-a_{1}}\right)-\theta_{i 2}
\end{array}\right)
$$

and

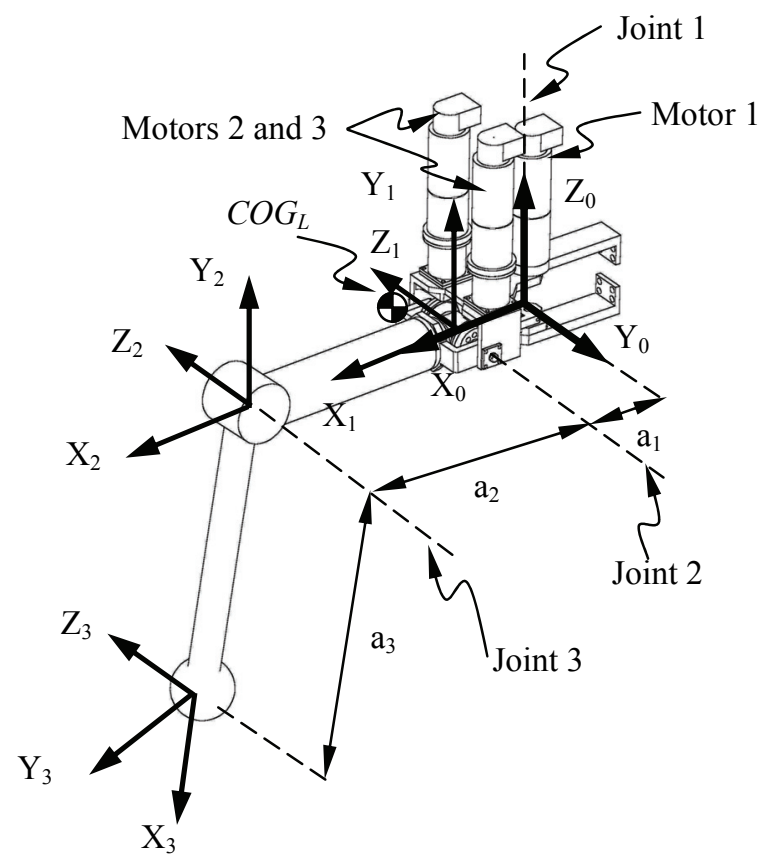

Fig. 10 SILO6 leg prototype, dimensions, and reference systems. 


$$
\begin{aligned}
A_{i} & =-z_{i} \\
B_{i} & =a_{1}-x_{i} \cos \theta_{i 1}+y_{i} \sin \theta_{i 1} \\
D_{i} & =\frac{2 a_{1}\left(x_{i} \cos \theta_{i 1}+y_{i} \sin \theta_{i 1}\right)+a_{3}^{2}-a_{2}^{2}-a_{1}^{2}-x_{i}^{2}-y_{i}^{2}-z_{i}^{2}}{2 a_{2}} .
\end{aligned}
$$

Joint reference frames and leg parameters are defined in Fig. 10. Link length values, $a_{i}$, are indicated in Table 1.

\section{Acknowledgement}

The authors would like to acknowledge the financial support of the Spanish Ministry of Education and Science through Grant DPI2004-05824.

\section{References}

Akizono, J., Iwasaki, M., Nemoto, T. and Asakura, O. 1989. Development on walking robot for underwater inspection. Proceedings of the International Conference on Advanced Robotics. Springer-Verlag. pp. 652-663.

Bares, J. E. and Wettergreen, D. S. 1999. Dante II: Technical description, results and lesson learned. The International Journal of Robotics Research 18(7): 621-649.

Bares, J. E. and Whittaker, W. L. 1993. Configuration of autonomous walkers for extreme terrain. The International Journal of Robotic Research 12(6):535-559.

Chen, J. S., Cheng, F. T., Yang, K. T., Kung, F. C. and Sun, Y. Y. 1999. Optimal force distribution in multilegged vehicles. Robotica 17: 159-172.

Gonzalez de Santos, P., Garcia, E., Estremera, J. and Armada, M. A. 2005a. DYLEMA: Using walking robots for landmine detection and location. International Journal of System Science 36(9): 545-558.

Gonzalez de Santos, P., Estremera, J. and Garcia, E. 2005b. Optimizing leg distribution around the body in walking robots. In Proceedings of the IEEE International Conference on Robotics and Automation, pp: 3218-3223, Barcelona, Spain.

Gonzalez de Santos, P., Armada, M. A. and Jiménez, M. A. 2000. Ship building with ROWER. IEEE Robotics and Automation Magazine 7(4):35-43.

Gonzalez de Santos, P., Jimenez, M.A. and Armada, M. 2002. "Improving the motion of walking machines by autonomous kinematic calibration", Autonomous Robots, 12(2): 187-199.

Gorinevsky, D. M. and Shneider, A. Y. 1990. Force control in locomotion of legged vehicles over rigid and soft surfaces. The International Journal of Robotics Research $9(2): 4-23$.

Grace, A. 1994. Optimization Toolbox for use with MATLAB. The Math Works Inc. 
Jiang, W. Y., Liu, A. M. and Howard, D. 2001. Foot-force distribution in legged robots. Proceedings of the 4th International Conference in Climbing and Walking Robots, pp. 331-338, Karlsruhe, Germany.

Klein, C. A. and Chung, T. S. 1987. Force interaction and allocation for the legs of a walking vehicle. IEEE Journal of Robotics and Automation RA-3(6):546-555.

Klein, C. A. and Kittivatcharapong, S. 1990. Optimal force distribution for the legs of a walking machine with friction cone constraints. IEEE Transactions on Robotics and Automation 6(1):73-85.

Kumar, V. R. and Waldron, K. J. 1988. Force distribution in closed kinematic chains. IEEE Journal of Robotics and Automation 4(6):657-664.

Kumar, V. R. and Waldron, K. J. 1990. Force distribution in walking vehicles. Journal of Mechanical Design 112: 90-99.

Nonami, K., Huang, Q. J., Komizo, D., Shimoi, N. and Uchida, H. 2000. Humanitarian mine detection six-legged walking robot. Proceedings of the 3rd International Conference on Climbing and Walking Robots, pp. 861-868, Madrid, Spain.

Pfeiffer, F. and Weidemann, H. J. 1991. Dynamics of the walking stick insect. IEEE Control Systems Magazine 11(2): 9-13.

Plustech-Oy. 2005. The walking forest machine concept. Available: http://agrosy.informatik.uni-kl.de/wmc/.

Salmi, S. and Halme, A. 1996. Implementing and testing a reasoning-based free gait algorithm in the six-legged walking machine MECANT. Control Engineering Practice 4(4): 487-492.

Schneider, A., Zeidis, I. and Zimmermann, K. 2000. Comparison of body shapes of walking machines in regards to stability margins. Proceedings of the 3rd International Conference on Climbing and Walking Robots, pp. 275-281, Madrid, Spain.

SILO6. 2006. The SILO6 Walking Robot. Available: http://www.iai.csic.es/users/silo6.

Song, S. M. and Waldron, K. J. 1989. MACHINES THAT WALK: The Adaptive Suspension Vehicle, The MIT Press Series in AI.

Waldron, K. J., Vohnout, V. J., Perry, A. and McGhee, R. B. 1984. Configuration design of the adaptive suspension vehicle. The International Journal of Robotic Research 3(2):3748 .

Zhou, D., Low, K.H. and Zielinska, T. 2000. An efficient foot-force distribution algorithm for quadruped walking robots. Robotica 18:403-413. 